\title{
LIGAMENTO CRUZADO ANTERIOR: PREVENCIÓN, REHABILITACIÓN PRE OPERATORIA Y POST OPERATORIA EN ATLETAS.
}

\author{
PREVIOUS CROSS LIGAMENT: PREVENTION, PRE-OPERATIVE REHABILITATION \\ AND POST OPERATORY AT ATHLETES.
}

\section{Carlos Miguel Entrena Yáñez \\ Fisioterapeuta \\ entrenacarlos@yahoo.com}

\section{Natalia Jimena Rincón Bolívar}

Fisioterapeuta

nataliarinconb@hotmail.com

\section{Ana Milena Rosas Quintero}

Fisioterapeuta

anamilenarosasquintero@gmail.com

\section{RESUMEN}

El ligamento cruzado anterior (LCA) es uno de los principales estabilizadores de la rodilla, porque influye en el control de esta e impide el desplazamiento anterior de la tibia con respecto al fémur, interviene de la misma manera, en controlar la rotación de la articulación femorotibial. Metodología: La lesión de ligamento cruzado anterior, es una de las principales afectaciones en atletas. Existen diversos medios para evaluar la integridad de dicho ligamento ya sean pruebas específicas de estabilidad (prueba de Lachman o cajón anterior) o pruebas clínicas (ecografía muscular, resonancia magnética). El objetivo de este artículo es evidenciar las nuevas tendencias fisioterapéuticas para la prevención y rehabilitación pre y post operatoria de los atletas con lesión de ligamento cruzado anterior, teniendo en cuenta los tiempos de cicatrización y la condición física del paciente, y el re integro a su gesto deportivo. Resultados: Es importante estructurar las metas del tratamiento para: prevenir la inestabilidad sintomática, restaurar la cinemática normal de la rodilla y prevenir la enfermedad articular degenerativa temprana, junto al adecuado y pertinente acondicionamiento muscular, con respecto a la fuerza y flexibilidad de miembro inferior.

Palabras clave: ligamento cruzado anterior, resonancia magnética, rehabilitación, tracción

\section{ABSTRACT}

The anterior cruciate ligament (ACL) is one of the main stabilizers of the knee, because it influences the control of this and prevents the anterior displacement of the tibia with respect to the femur, intervenes in the same way, in controlling the rotation of the joint femorotibial. Methodology: The anterior cruciate ligament injury is one of the main affectations in athletes. There are several means to evaluate the integrity of said ligament either specific stability tests (Lachman test or anterior drawer) or clinical tests (muscle ultrasound, magnetic resonance). The objective of this article is to 
demonstrate the new physiotherapeutic tendencies for the prevention and rehabilitation, both pre- and post-operatively, of athletes with anterior cruciate ligament injury, taking into account the healing times and the physical condition of the patient, and the complete reintegration of sporting gesture. Results: It is important to structure treatment goals to: prevent symptomatic instability, restore normal knee kinematics and prevent early degenerative joint disease, together with adequate and appropriate muscle conditioning, with respect to lower limb strength and flexibility.

Key words: anterior cruciate ligament, magnetic resonance, rehabilitation, traction.

\section{INTRODUCCIÓN}

El ligamento cruzado anterior (LCA) se ubican en la rodilla, en donde su nombre proviene del cruce en forma de " $X$ " con el ligamento cruzado posterior en la zona media de la rodilla y pasa por delante de éste (Yanguas, J., et al., 2011). Es el principal estabilizador de la rodilla, sin él la rodilla es incapaz de aguantar el peso corporal en movimiento o carga. Además es indispensable para la rotación de rodilla y evita que la tibia se desplace hacia delante en relación al fémur (Márquez, J., 2011). La lesión del LCA es una de las lesiones más común en la articulación de la rodilla (Del Bel, M., et al., 2017) y es una de las más reportadas en los individuos activos (Agel, J., et al., 2016) y es más frecuente en mujeres que en hombres, debido a la laxitud de sus ligamentos (Yanguas, J., et al., 2011) (Wordeman, S., et al., 2014). También pueden ser consideradas como devastadoras para la carrera del atleta y de su calidad de vida a nivel general (6). Debido a que la rotura del LCA rara vez se produce de manera aislada; suele ir acompañada de lesiones de estructuras adyacentes como por ejemplo el ligamento lateral interno, meniscos, lesiones óseas etc.; en la mayoría de los casos. Esto genera que su lesión retarde el tiempo de vuelta al juego (Yanguas, J., et al., 2011) (Wordeman, S., et al., 2014). Ahora bien, las lesiones de LCA generalmente requieren una cirugía invasiva y de un proceso de rehabilitación y también conducen a una mayor probabilidad de osteoartritis de rodilla de inicio temprano (Hewett, T., et al., 2015).

Cuando se presenta una rotura del LCA se pueden encontrar dos tipos de pacientes: los que toleran y los que no toleran la ruptura del LCA. Los tolerantes son aquellos pacientes que realizan de forma asintomática todas las actividades, que incluyen las deportivas que realizaban antes de la ruptura del ligamento. Por otro lado, los no tolerantes son aquellos que presentan síntomas relacionados con la inestabilidad articular y por lo tanto necesitan de reconstrucción (Del Bel, M., et al., 2017), para regresar a sus actividades de la vida diaria. Para la reconstrucción de LCA se han descrito muchas técnicas quirúrgicas donde se emplean varios tipos de injertos $y$ fijaciones, tanto a nivel tibial como femoral. En la actualidad, el injerto con tendón rotuliano (técnica HTH) y la técnica del semitendinoso son las más empleadas (Del Gordo, D., 2011) (Chahla, J., et al., 2015). Sin embargo, esta última técnica se está practicando con más frecuencia, debido a que existe una menor morbilidad en la zona donante de donde se obtienen el neoligamento (Álvarez, A., et al., 2015). 


\section{BIOMECÁNICA}

El LCA es la primera restricción para el desplazamiento anterior de la tibia. El ligamento cruzado posterior (LCP) y el LCA determinan la combinación de deslizamiento y rodamiento entre la tibia y el fémur que caracteriza la cinemática de la rodilla normal. Todo esto es gracias a que el LCA posee una serie de fibras que están tensas en diferentes posiciones de la rodilla (Wordeman, S., et al. 2014) (Dienst, M., et al., 2002). Se ha encontrado que las fibras más isométricas son las que pertenecen a la banda antero medial (AM); la mayor parte del ligamento está tensa cuando la rodilla está extendida y relativamente laxa cuando está en flexión. De esta manera, la deficiencia del LCA no solo produce episodios de inestabilidad, sino también, una alteración de la mecánica articular, lo cual puede contribuir a los cambios degenerativos de dicho ligamento (Hewett, T., et al., 2015). Es importante plantear las metas del tratamiento para: prevenir la inestabilidad sintomática, restaurar la cinemática normal de la rodilla y prevenir la enfermedad articular degenerativa temprana (Hewett, T., et al., 2015) (Dienst, M., et al., 2002).

Cuando hay una lesión del LCA la tibia se puede subluxar anteriormente con los signos clínicos correspondientes, pero también puede haber cambios sutiles de la función articular, a saber: desplazamientos en la localización del centro de rotación instantáneo, es decir, para cada ángulo de movimiento, haciendo que los vectores de velocidad, que normalmente son paralelos a la superficie articular, dejen de serlo; se producen así fuerzas compresivas a través de la articulación que pueden explicar la enfermedad articular degenerativa acelerada que frecuentemente acompaña las lesiones de este ligamento (Álvarez, A., et al., 2015).

\section{MECANISMO DE LESIÓN}

La mayor incidencia de lesiones del LCA se produce como consecuencia de traumatismos indirectos sobre la rodilla durante la práctica deportiva en pacientes jóvenes, frecuentemente no son diagnosticadas en el momento inicial. Los síntomas más frecuentes tras la lesión del LCA son dolor, tumefacción articular leve y sensación de inestabilidad de rodilla, principalmente en actividades de torsiónrecorte-desaceleración. Cuando se genera la ruptura del ligamento cruzado anterior, el atleta siente como la rodilla no presenta tope y refiere que "se le va" (Yanguas, J., et al., 2011) (Márquez, J., 2011). Por lo general se lesiona cuando el atleta realiza una rotación de la pierna y al mismo tiempo se produce una fuerte carga en sentido descendente. Ejemplos de este movimiento serían las caídas por salto, donde la rodilla se va hacia delante de manera brusca. También, se produciría en los cambios de direcciones, porque como se ha mencionado se aplica una fuerza en dirección descendente y la rodilla suele estar rotada para iniciar la carrera en sentido contrario (Yanguas, J., et al., 2011).

\section{FACTORES DE RIESGO}

Existen dos factores principales de riesgo que contribuyen a la lesión de LCA: la biomecánica del movimiento (cinemática, cinética, la magnitud de la activación muscular y la producción de fuerza), y la insuficiencia neuromuscular. Diversos 
estudios han comprobado que la deformación del LCA aumenta con fuerzas de compresión tibial anterior, femorotibial y en la rotación combinada de rodilla, otro factor es cuando se genera una desaceleración de manera súbita y a la vez se cambia la dirección al correr o cuando se aterriza de un salto y el atleta cae y la pierna se va en valgo (Márquez, J., 2011). Por otro lado, la insuficiencia neuromuscular se ve reflejada en la reacción de la línea de base, la velocidad de procesamiento de un movimiento y la percepción visual- espacial en atletas. Aunque en este tipo de lesiones asimismo entran a influir la alineación estructural de las extremidades articulación de la rodilla, su laxitud, composición corporal, sexo, entre otros factores (Yanguas, J., et al., 2011) (Shultz, S., et al., 2015).

\section{PREVENCIÓN}

La lesión de LCA en atletas como se mencionó anteriormente está directamente relacionada a las debilidades en el sistema neuromuscular y los desbalances musculares (Márquez, J., 2011). Por otra parte, en pacientes que ya han sido intervenidos quirúrgicamente, se genera una reincidencia en esta lesión. En los atletas, la mayor parte de las segundas lesiones del LCA ocurren dentro de los primeros 2 años de regreso al deporte. Presentando alteraciones funcionales significativas y déficits en el control neuromuscular (Lang, P., et al., 2017) (Wonderman, S., et al., 2016).

Para su prevención es importante implementar ejercicios donde se trabaje la cadena muscular de miembros inferiores de manera equitativa, a saber, que se busque un balance muscular para evitar compensaciones o alteraciones en los rangos de movilidad (ROM) (Márquez, J., 2011) (Lang, P., et al., 2017). Otro aspecto importante a tratar es la flexibilidad muscular y la propiocepción (Paús, V., et al., 1998) (Souissi, S., et al., 2013), ya que de esta manera, se entrena a los elementos de estabilización articular (capsula articular, tendones y ligamentos) para que tengan una mayor fuerza tensil y velocidad de reacción con respecto a la variación de movimientos (Wonderman, S., et al., 2016).

\section{REHABILITACIÓN PRE OPERATORIA}

Antes de la cirugía reconstructiva de LCA debe hacerse hincapié en el establecimiento de un patrón de marcha normal y un rango de movimiento activo de al menos $0^{\circ}$ a $90^{\circ}$, de hecho, que el atleta tenga una extensión completa y el proceso de inflamación se encuentre controlado (Malempati, C., et al., 2015). Igualmente, el entrenamiento neuromuscular y el fortalecimiento muscular para evitar que en el periodo post operatorio haya un compromiso mayor, evidenciado en la atrofia muscular, en la contracción muscular (reclutamiento de fibras musculares) y en la fuerza muscular principalmente del miembro afectado (Malempati, C., et al., 2015).).

\section{REHABILITACIÓN POST OPERATORIA}

Los enfoques de rehabilitación de LCA actuales hacen hincapié en la importancia del movimiento inmediato y levantamiento de peso temprano, ejercicios de los músculos inmediatos, ejercicios cinéticos de la cadena, actividades funcionales, principios y un retorno más rápido a los deportes. De la 
misma forma se deben implementar ejercicios de control propioceptivo y neuromuscular para que los atletas de alto nivel puedan recuperar la estabilidad de la articulación dinámica y funcional necesaria en la competición atlética (Lang, P., et al., 2017) (Almeida, G., et al., 2014). Es significativo al momento de realizar la planificación del entrenamiento específico, tener en cuenta las condiciones físicas del atleta y su gesto deportivo. En las primeras 4 semanas después de la cirugía, los objetivos durante este período son para minimizar el dolor y la inflamación mediante el manejo de medios terapéuticos (termoterapia, crioterapia, magnetoterapia, etc.) (Abush, T., et al., 2012), ganar rangos de movilidad articular haciendo énfasis en la extensión, esto es importante lograrlo en el primer mes post quirúrgico mediante la movilidad activa asistida, en muchos casos cuando el atleta presenta una disminución de ROM en extensión es necesario el uso de la técnica de tracción (Adams, D., et al., 2012). Por un lado, en lo que respecta a la flexión, se debe ir ganando de manera progresiva teniendo en cuenta el tipo de técnica quirúrgica, fortalecer tanto la musculatura flexora y extensora de rodilla de manera progresiva, respetando los tiempos de cicatrización de las estructuras indemnes y trabajar de manera específica en diversos ejercicios de propiocepción para reeducar al ligamento y de esta manera favorecer las repuestas automáticas y reflejas del mismo ante los movimientos (17).
Retomando, en la fase de fortalecimiento de la rehabilitación de un postoperatorio (POP) de LCA, es importante primero que la amplitud del movimiento y la movilización de la rótula se debe alcanzar lo antes posible ya que de esta manera se evitan adherencias en el tendón rotuliano, segundo esta fase generalmente se implementa posterior a la semana 4 hasta el mes 6 y puede ser prolongado más tiempo de ser necesario (Malempati, C., et al., 2015).). La progresión de la misma está sujeta a que el atleta no presente dolor articular ni inflamación a corto plazo posterior a los ejercicios, de ser así se debe suspender los ejercicios entre 6 a 12 horas en general.

En esta fase, el objetivo principal debe ser la técnica apropiada y evitar la sustitución mediante mecanismos de compensación. En cuanto al manejo de pesos, estos se deben aumentarse gradualmente junto con movimientos multidireccionales los cuales se pueden incorporar para imitar la actividad funcional y atlética (Malempati, C., et al., 2015).).

A continuación se expone una revisión sistemática basada en evidencia correspondiente al periodo de enero a noviembre del 2015 realizada en el Centro Médico Deportivo MET, establecida para el proceso de rehabilitación tras POP de ligamento cruzado anterior. En las siguientes tablas se encontraran puntos guía basados en evidencia para el tratamiento de un posoperatorio de un LCA: 


\begin{tabular}{|c|c|c|c|c|}
\hline $\begin{array}{l}\text { TIEMPO } \\
\text { EVOLUCIÓN }\end{array}$ & $\begin{array}{l}\text { DISMINUIR DOLOR E } \\
\text { INFLAMACIÓN Y } \\
\text { FAVORECER } \\
\text { PROCESO DE } \\
\text { CICATRIZACIÓN }\end{array}$ & $\begin{array}{c}\text { GANAR } \\
\text { RANGO DE MOVIMIENTO } \\
\text { (ROM) }\end{array}$ & $\begin{array}{l}\text { REEDUCAR YIO AUMENTAR } \\
\text { LA FUERZA MUSCULAR }\end{array}$ & $\begin{array}{l}\text { REINTEGRAR } \\
\text { PACIENTE } \\
\text { ACTIVIDADES } \\
\text { DEPORTIVAS }\end{array}$ \\
\hline SEMANA 0-4 & $\begin{array}{l}\text { - Crioterapia: aplicar } \\
\text { hielo } 7 \text { minutos sobre } \\
\text { la rodilla lesionada } \\
\text { para disminuir el dolor } \\
\text { y la inflamación. } \\
\text { (19) (20)(22)(23) (24) } \\
\text { (25) (26) (27). } \\
\text { - Elevación del miembro } \\
\text { inferior para controlar } \\
\text { inflamación o edema. } \\
\text { (25)(28). } \\
\text { - Termoterapia o calor } \\
\text { húmedo. } \\
\text { - Ejercicios de bombeo } \\
\text { circulatorio(29) (30). } \\
\text { - Magnetoterapia: modo } \\
\text { pulsátil y 75Hz (31). } \\
\text { - Técnica de RICE } \\
\text { (Reposo, Hielo, } \\
\text { Compresión). (19)(29). } \\
\text { - Estimulación eléctrica } \\
\text { del cuádriceps } \\
\text { (24) (27) (15). } \\
\text { - Movilización } \\
\text { cicatriz (20). de } \\
\text { - Movilización de la } \\
\text { rótula (20). } \\
\text { - Vendaje compresivo } \\
\text { (25). }\end{array}$ & $\begin{array}{l}\text { - Ejercicios de movilidad } \\
\text { articular en cadena } \\
\text { cinética cerrada. (19) } \\
\text { ROM activo } 0^{\circ}-90^{\circ} \text { flexión } \\
\text { - extensión (22) (23). } \\
\text { Progresando a 0-110 según } \\
\text { tolerancia (20). } \\
\text { - Progresar hasta lograr a } \\
\text { flexo-extensión completa } \\
\text { en este periodo de } \\
\text { tiempo (32) (25) (15). } \\
\text { - Al } 10 \text { día se empieza a } \\
\text { ganar la extensión de la } \\
\text { rodilla con la técnica de } \\
\text { tracción (21). } \\
\text { - Flexibilidad a tolerancia de } \\
\text { los músculos } \\
\text { isquiotibiales. (33). } \\
\text { - Movilización de la } \\
\text { rótula (34) (31). }\end{array}$ & $\begin{array}{l}\text { - Ejercicios isométricos de } \\
\text { cuádriceps, aductores e } \\
\text { isquiotibiales para prevenir atrofia } \\
\text { muscular (31) (35) (36). } \\
\text { - Ejercicios isotónicos de miembro } \\
\text { inferior en cadena cinética abierta } \\
\text { (CCA) (24) (35) (31) (35). } \\
\text { - Ejercicio de contracción } \\
\text { simultánea de los extensores y } \\
\text { músculos flexores en } 20^{\circ} \text { de } \\
\text { flexión (33). } \\
\text { - Ejercicio de fortalecimiento para } \\
\text { plantiflexores y dorsiflexores de } \\
\text { cuello de pie con theraband (36). } \\
\text { - Ejercicios excéntricos de } \\
\text { isquiotibiales en CCA (4a semana } \\
\text { en adelante). (19) (20) (25) (31) } \\
\text { (35) (37). }\end{array}$ & \\
\hline SEMANA 5-8 & $\begin{array}{l}\text { - Ejercicios de bombeo } \\
\text { de cuello de pie (30). } \\
\text { - Continuidad de la } \\
\text { restricción de la } \\
\text { actividad deportiva } \\
\text { (38). } \\
\text { - Crioterapia, electro- } \\
\text { estimulación } \\
\text { neuromuscular (27). }\end{array}$ & $\begin{array}{l}\text { Continuar con el proceso } \\
\text { anterior. }\end{array}$ & $\begin{array}{l}\text { - Entrenamiento isocinético (24). } \\
\text { - Ejercicios de cadena cinética } \\
\text { cerrada } 20^{\circ} \text { a } 90^{\circ} \text { de manera } \\
\text { progresiva (29) (32) (39). } \\
\text { - Natación (opcional). Superior al } \\
\text { mes o por indicación médica } \\
\text { (23). } \\
\text { - Fortalecimiento } \\
\text { isquiotibiales, cadena cinética } \\
\text { cerrada, ejercicios excéntricos } \\
\text { (24) (33) (25). } \\
\text { - Ejercicios isométricos y } \\
\text { ejercicios isotónicos de } \\
\text { cuádriceps, aductores e } \\
\text { isquiotibiales para la activación } \\
\text { muscular del mismo, mantener } \\
\text { trofismo muscular (31)(35) } \\
\text { (39). }\end{array}$ & $\begin{array}{l}\text { Continuar con lo } \\
\text { estipulado } \\
\text { anteriormente. }\end{array}$ \\
\hline
\end{tabular}




\begin{tabular}{|c|c|c|c|c|}
\hline $\begin{array}{l}\text { SEMANA 9- } \\
12\end{array}$ & \multirow{3}{*}{ - Crioterapia (27). } & \multirow{3}{*}{$\begin{array}{l}\text { - } \text { ROM de rodilla } \\
\text { completo(15)(31) (34). } \\
\text { - Reincorporación a } \\
\text { actividades deportivas de } \\
\text { baja intensidad (32). } \\
\text { - Ejercicios de flexibilidad, } \\
\text { natación, montar en } \\
\text { bicicleta estática con leve } \\
\text { resistencia (33). } \\
\text { - Subir escaleras: frontal y } \\
\text { laterales (25). }\end{array}$} & $\begin{array}{l}\text { - Según el progreso de los } \\
\text { ejercicios se aumenta la } \\
\text { intensidad y duración (33)(34). } \\
\text { - Deben iniciarse ejercicios de } \\
\text { potenciación en carga: } \\
\text { excéntricos cuádriceps (3 } \\
\text { meses) en cadena cinética } \\
\text { cerrada } 90^{\circ} \text { de flexión (24) (35) } \\
\text { (37). } \\
\text { - Ejercicios para fortalecimiento } \\
\text { de isquiotibiales (23) (33) (27). } \\
\text { - Entrenamiento de Core (33). }\end{array}$ & \\
\hline MES 3-4 & & & $\begin{array}{l}\text { - Sentadilla mantenida a } 90^{\circ} \text { y } \\
\text { combinación de ejercicios en } \\
\text { cadena cinética cerrada } \\
\text { (CCC). (34) (37) } \\
\text { - Continuar con el } \\
\text { fortalecimiento muscular los } \\
\text { cuádriceps, isquiotibiales, } \\
\text { gemelos(26) y estructuras } \\
\text { indemnes. (23) (33). } \\
\text { - Ejercicios polimétricos (27). } \\
\text { - Entrenamiento de Core (33). } \\
\text { - Aumento de la intensidad y la } \\
\text { complejidad del fortalecimiento } \\
\text { funcional (34). }\end{array}$ & $\begin{array}{l}\text { - Entrenar } \\
\text { gesto } \\
\text { específico del } \\
\text { deporte (39). }\end{array}$ \\
\hline $\begin{array}{l}\text { Mayor a } 4 \\
\text { meses }\end{array}$ & & & $\begin{array}{l}\text { - Aumento de la intensidad y la } \\
\text { complejidad de fortalecimiento } \\
\text { funcional sentadillas cuclillas } \\
\text { con peso (37). } \\
\text { - Al } 5^{0} \text { mes ambos injertos son } \\
\text { capaces de soportar alrededor } \\
\text { del } 80 \% \text { de su máxima } \\
\text { resistencia tensil, lo que es } \\
\text { muy similar a la resistencia } \\
\text { tensil del ligamento cruzado } \\
\text { original. Si clínicamente el } \\
\text { paciente está apto, se pueden } \\
\text { reiniciar actividades en campo } \\
\text { de forma progresiva (38). } \\
\text { - Gesto específico del deporte } \\
\text { (39). } \\
\text { - Al año: se retorna a la } \\
\text { actividad deportiva plena con los } \\
\text { criterios: 1) balance articular } \\
\text { completo; 2) no derrame; 3) } \\
\text { rodilla estable, 4) haber } \\
\text { completado el programa de } \\
\text { agilidad. (20) (37). }\end{array}$ & $\begin{array}{l}\text { - Al año se } \\
\text { retorna a la } \\
\text { actividad } \\
\text { deportiva plena } \\
(20)(37) \text {. }\end{array}$ \\
\hline
\end{tabular}




\begin{tabular}{|c|c|c|c|}
\hline $\begin{array}{c}\text { TIEMPO } \\
\text { EVOLUCIÓN }\end{array}$ & REEDUCAR PROPIOCEPCIÓN & REENTRENAR LA MARCHA & $\begin{array}{c}\text { REENTRENAR CAPACIDAD } \\
\text { AERÓBICA }\end{array}$ \\
\hline SEMANA 0-4 & $\begin{array}{l}\text { - Ejercicios de balance estático y } \\
\text { propiocepción bipodal (23). }\end{array}$ & $\begin{array}{l}\text { - La marcha es permitida a las } 48 \\
\text { horas de la cirugía (cuando se } \\
\text { retiran los drenajes) con empleo } \\
\text { de dos muletas (15). } \\
\text { - } \quad \text { Reentrenamiento de marcha con } \\
2 \text { muletas (33). } \\
\text { - Deambulación, utilizando } \\
\text { muletas, con apoyo a tolerancia } \\
\text { del miembro afectado. (19). } \\
\text { - Marcha a } 3 \text { puntos con } \\
\text { muletas (8). } \\
\text { - La marcha y las escaleras } \\
\text { generan aproximadamente } 300 \\
\text { newton de tensión sobre el injerto } \\
\text { y por ende se pueden iniciar en } \\
\text { forma precoz, según la tolerancia } \\
\text { del paciente (38). } \\
\text { Marcha con retiro gradual de } \\
\text { muletas (8). } \\
\text { Cuarta semana el paciente } \\
\text { comenzara a caminar sin ningún } \\
\text { tipo de ayuda (15) para el soporte } \\
\text { de peso completo (36). }\end{array}$ & - Cicla estática (25) \\
\hline SEMANA 5-8 & $\begin{array}{l}\text { - } 4 \text { o } 5 \text { semana: entrenamiento de } \\
\text { la sensibilidad propioceptiva } \\
\text { (equilibrio estático, equilibrio } \\
\text { dinámico sobre balanza de } \\
\text { Freeman.(34) (38) (39). } \\
\text { - Entrenamiento propioceptivo, } \\
\text { entrenamiento } \\
\text { funcional.(20)(23) (33). } \\
\text { - Trabajo de coordinación y } \\
\text { equilibrio. (39) (15). }\end{array}$ & $\begin{array}{l}\text { - Entrenamiento de marcha para } \\
\text { adelante y para atrás con cambios } \\
\text { de dirección utilizando } \\
\text { conos.(24)(29)(20). } \\
\text { - Entrenamiento funcional y de } \\
\text { reeducación de la marcha } \\
(15)(27)(29)(33) \text {. }\end{array}$ & $\begin{array}{l}\text { - Uso de bicicleta(37). } \\
\text { - Ejercicios aeróbicos } \\
\text { (miembros superiores) (19). }\end{array}$ \\
\hline $\begin{array}{l}\text { SEMANA 9- } \\
12\end{array}$ & $\begin{array}{l}\text { - Trabajo de coordinación y } \\
\text { equilibrio (39). } \\
\text { - Ejercicios de equilibrio } \\
\text { combinados con entrenamiento } \\
\text { funcional (27). }\end{array}$ & $\begin{array}{l}\text { - Caminar 10-20 minutos (40). } \\
\text { - Entrenamiento funcional y de } \\
\text { reeducación de la marcha (29) (27). }\end{array}$ & $\begin{array}{l}\text { - Trote ligero en una dirección } \\
\text { sobre superficie uniforme. } \\
\text { - Intensificar ejercicios de } \\
\text { agilidad (23) (27). } \\
\text { - Cicla estática con } \\
\text { resistencia de manera } \\
\text { progresiva (25) (39) (37). } \\
\text { - Traslado al gimnasio si se } \\
\text { cumplen con los objetivos (34). } \\
\text { - Ejercicios aeróbicos (19). }\end{array}$ \\
\hline MES 3-4 & $\begin{array}{l}\text { - Progresión en ejercicios de trabajo } \\
\text { de propiocepción (33) (37) } \\
\text { - Entrenamiento propioceptivo } \\
\text { combinado con entrenamiento } \\
\text { funcional, de acuerdo al deporte } \\
\text { que practique el deportista (27). }\end{array}$ & $\begin{array}{l}\text { - Caminar 10-20 minutos. (40) } \\
\text { - Marcha (hacia atrás, zigzag, puntas } \\
\text { de pie, etc.) (15). } \\
\text { - Trote progresivo (19). } \\
\text { - Al iniciar los } 4 \text { meses se puede } \\
\text { iniciar con el correr (37). }\end{array}$ & $\begin{array}{l}\text { - Entrenamiento funcional } \\
\text { según deporte del paciente } \\
\text { (baja intensidad) } \\
\text { Carrera en una dirección } \\
\text { sobre cualquier superficie. } \\
\text { Iniciar actividades de carrera } \\
\text { con trote, giro y cambios de } \\
\text { dirección (23) (27). } \\
\text { - Trote en banda sin fin (9)(23). } \\
\text { - Ejercicios aeróbicos en } \\
\text { bicicleta(19)(25)(29)(37). }\end{array}$ \\
\hline
\end{tabular}




\begin{tabular}{|c|c|c|c|}
\hline & & & $\begin{array}{l}\text { - Entrenamiento para reanudar } \\
\text { la actividad deportiva previa } \\
\text { (24). }\end{array}$ \\
\hline MES 4-5 & $\begin{array}{l}\text { - Estabilidad a pierna lesionada } \\
\text { sobre disco inestable luego sobre } \\
\text { plataforma rectangular } \\
\text { inestable (16). } \\
\text { - Entrenamiento propioceptivo, } \\
\text { entrenamiento funcional } \\
\text { especifico del deporte (27). } \\
\text { - Ejercicios de coordinación y } \\
\text { equilibrio, resistencia (39). }\end{array}$ & $\begin{array}{l}\text { - Trote progresivo (19). } \\
\text { - Correr (37). } \\
\text { - Marcha funcional (27) (19). } \\
\text { - Se inicia el entrenamiento para } \\
\text { incorporarse a la actividad } \\
\text { deportiva (16) (24). }\end{array}$ & $\begin{array}{l}\text { - Actividades en el terreno de } \\
\text { juego (24). } \\
\text { - Ejercicios con mayor } \\
\text { resistencia (39). }\end{array}$ \\
\hline
\end{tabular}

\section{METODOLOGÍA}

Se realizó una revisión de 40 artículos en Scielo, Medline, Elsevier, NCBI, Ovid y Proquest publicados entre el año 2015 y 2017, en donde 10 artículos fueron seleccionados y nexados junto con la revisión sistemática ejecutada en el año 2015 en el Centro Médico Deportivo MET; basada en el proceso de rehabilitación tras el postoperatorio del ligamento cruzado anterior en el deportista. Posterior a esto, se realizó un análisis y comparación de la intervención tanto en su enfoque preventivo, en la fase pre operatoria, y finalmente, en la postoperatoria de la reconstrucción del LCA, teniendo en cuenta los tiempos de lesión y cicatrización.

\section{CONCLUSIONES}

El enfoque preventivo de la lesión de LCA se basa en el entrenamiento neuromuscular mediante ejercicios de propiocepción aplicados al gesto deportivo de cada atleta, junto al correcto acondicionamiento muscular con respecto a la fuerza $y$ flexibilidad de miembro inferior.

En la lesión de LCA tanto en la etapa de rehabilitación preoperatoria como post operatoria es indispensable realizar la intervención del deportista bajo los principios de entrenamiento y los tiempos de cicatrización de los tejidos. Así mismo, teniendo en cuenta el dolor, el rango de movimiento, la flexibilidad, la fuerza muscular y el equilibrio, balance y propiocepción, para posteriormente, el reintegro al campo y a su reincorporación deportiva, y finalmente, evitar la reincidencia de la lesión. 


\section{REFERENCIAS BIBLIOGRÁFICAS}

Yanguas, J., Pérez, T., Cortés de Olano, C. (2011). Lesión del ligamento cruzado anterior en fútbol femenino. Estudio epidemiológico de tres temporadas. Apunt Med l'Esport. 46(171):137-43.

Márquez, J. (2008). Márquez Arabia WH. Lesiones del ligamento cruzado anterior de la rodilla. Acta Ortopédica Mex.28(1):57-67.

Del Bel, M,, Fairfax, A., Jones M., Steele, K., Landry, S. (2017). Effect of limb dominance and sex on neuromuscular activation patterns in athletes under 12 performing unanticipated sidecuts. J Electromyogr Kinesiol. 36:65-72.

Agel, J., Rockwood, T., Klossner, D. (2016). Collegiate ACL Injury Rates Across 15 Sports: National Collegiate Athletic Association Injury Surveillance System Data Update (2004-2005 Through 2012-2013). Clin J Sport Med Off J Can Acad Sport Med. 26:518-23.

Wordeman, S., Hewett, T., Muñoz, D., Espí-López, V., Dienst, M., Burks, R., et al. (2014). Anatomy and biomechanics of the anterior cruciate ligament. Rev Arch Médico Camaguey [Internet]. 24(1):7-11. Available from: http://bmcmusculoskeletdisord.biomedcentral.com/articles/10.1186/1471-2474-14-108

Button, K., Roos, P., Van Deursen, V. (2014). Activity progression for anterior cruciate ligament injured individuals. Clin Biomech. 29(2):206-12.

Hewett, T., Myer, G., Kiefer, A., Ford, K. (2015). Longitudinal Increases in Knee Abduction Moments in Females during Adolescent Growth. Med Sci Sports Exerc. 47(12):2579-85.

Del Gordo, D. (2011). Reconstrucción artroscópica del ligamento cruzado anterior con injerto autólogo de semitendinoso cuádruple. Duazary. 8(1):34-41.

Chahla, J., Arroquy, D., Gómez, G., Pérez, G., Chahla, J. (2015). Uso de Aloinjerto vs . Autoinjerto en la Reconstrucción del Ligamento Cruzado Anterior: Seguimiento a 4 Años. Artroscopia. 22(2):51-5.

Álvarez, A., García, Y. (2015). Lesiones del ligamento cruzado anterior [Internet]. Vol. 19, Revista Archivo Médico Camaguey. Available from: http://scielo.sld.cu/scielo.php?script=sci_arttext\&pid=S1025-02552015000100014

Dienst, M., Burks, R., Greis, P. (2002). Anatomy and biomechanics of the anterior cruciate ligament. Orthop Clin. 33:605-20.

Shultz, S., Schmitz, R., Benjaminse, A., Collins, M., Ford, K., Kulas, A. (2015). ACL research retreat VII: An update on anterior cruciate ligament injury risk factor identification, screening, and prevention. J Athl Train. 50(10):1076-93.

Lang, P., Sugimoto, D., Micheli, L. (2017). Prevention, treatment, and rehabilitation of anterior cruciate ligament injuries in children. Open Access J Sport Med [Internet]. Volume 8:133-41. Available from: https://www.dovepress.com/prevention-treatment-and-rehabilitation-ofanterior-cruciate-ligament--peer-reviewed-article-OAJSM

Wordeman, S., Hewett, T. (2016). Research-based and clinical considerations for effective neuromuscular training to prevent second Anterior Cruciate Ligament injury. Oper Tech Sports Med. 24(1):7-11.

Paús, V. Esper, A. (1998). La periodización y la planificación de la rehabilitación del ligamento 
cruzado anterior. Rev Digit Educ Física.3(12).

Souissi, S., Wong, D., Dellal, A., Croisier, J., Ellouze, Z., Chamari, K. (2013). Mejora del Rendimiento Funcional y Potencia Muscular entre el 4to y 6to Mes Luego de una Reconstrucción del Ligamento Cruzado Anterior [Internet]. PubliCE Premium. Available from: https://g-se.com/mejora-del-rendimiento-funcional-y-potencia-muscular-entre-el-4toy-6to-mes-luego-de-una-reconstruccion-del-ligamento-cruzado-anterior-1503-sa$557 \mathrm{cfb} 2721 \mathrm{fb} 45$

Malempati, C., Jurjans, J., Noehren, B., Ireland, M., Johnson, D. (2015). Current Rehabilitation Concepts for Anterior Cruciate Ligament Surgery in Athletes. Orthopedics. 38(11):689-96.

Leão, G., De Oliveira, G., Marques, A. (2014). Physical therapy in the conservative treatment for anterior cruciate ligament rupture followed by contralateral rupture: case report. Fisioter e Pesqui. 21(2):186-92.

Abush, S., Atri, J. (2012). Reconstrucción del ligamento cruzado anterior en pacientes mayores de 41 años. Resultados utilizando aloinjerto de hueso tendón rotuliao. An Med Mex. 57(2):104-11.

Muñoz, D., Espí-López, V. (2014). Proceso de recuperación funcional tras lesión del ligamento cruzado anterior. Revisión bibiográfica. Fisio Divulg. 2(3):3-12.

Adams, D., Logerstedt, D., Hunter-Giordano, A., Axe, M., Snyder-Mackler, L. (2012). Current Concepts for Anterior Cruciate Ligament Reconstruction: A Criterion-Based Rehabilitation Progression. J Orthop Sport Phys Ther.42(7):601-14.

Jarmuziewicz, A., Snela, S., Bielecki, A., Dudek, J., Bazarnik-Mucha, K. (2014). Dynamics of the increase in strength and girth of quadriceps muscle after the reconstruction of anterior cruciate ligament. Polish Orthop Traumatol. 79:71-6.

Figueroa, D., Calvo, R., Anastasiadis, Z., Vaisman, A., Scheu, M. (2013). ¿En Cuánto Tiempo Recuperan la Movilidad Articular los Pacientes Operados de Reconstrucción de Ligamento Cruzado Anterior? Artroscopia. 20(1):1-6.

Quelard, B., Rachet, O., Sonnery-Cottet, B., Chambat, P. (2010). Rehabilitación postoperatoria de los injertos del ligamento cruzado anterior. Elsevier, editor. Elsevier Masson. 16 p.

Uçar, M., Koca, I., Eroglu, M., Eroglu, S., Sarp, U., Arik, H., et al. (2014). Evaluation of open and closed kinetic chain exercises in rehabilitation following anterior cruciate ligament reconstruction. J Phys Ther Sci. 2014;26(12):1875-8.

Pereira, M., De Souza, N, Da Rosa, E. Alfonso, J., Grignet, R., Frèz, A. (2012). Tratamento fisioterapêutico após reconstrução do Ligamento Cruzado Anterior. Acta Ortopédica Bras. 20(6):372-5.

Dragicevic-Cvjetkovic, D., Jandric, S., Bijeljac, S., Palija, S., Manojlovic, S., Talic, G. (2014). The effects of rehabilitation protocol on functional recovery after Anterior Cruciate Ligament reconstruction. Med Arh. 68(5):350-2.

Dambros, C., Martimbianco, A. Polachini, L., Lahoz, G., Chamlian, T., Cohen, M. (2012). Effectiveness of cryotherapy after anterior cruciate ligament reconstruction. Acta Ortop Bras. 20(5):285-90.

Yabroudi, M., Irrgang, J. (2013) Rehabilitation and Return to Play After Anatomic Anterior Cruciate Ligament Reconstruction. Clin Sports Med. 32(1):165-75.

Lee, J., Kim, J., Park, G. (2013). Effect of 12 weeks of accelerated rehabilitation exercise on 
muscle function of patients with ACL reconstruction of the knee joint. J Phys Ther Sci. 25(12):1595-9.

Kruse, L., Gray, B., Wright, R. (2012). Rehabilitation After Anterior Cruciate Ligament Reconstruction. Asystematic Review. J Bone Jt Surg. 2012;94(19):1737-48.

Brewer, B., Cornelius, A., Raalte, J., Tennen, H., Armeli, S. (2013). Predictors of adherence to home rehabilitation exercises following Anterior Cruciate Ligament reconstruction. Rehabili psychol. 58(1):64-72.

Saka, T. (2014). Principles of postoperative anterior cruciate ligament rehabilitation. World J Orthop. 5(4):450-9.

Adams, D., Logerstedt, D., Hunter-Giordano, A., Axe, J., Snyder-Mackler, L. (2013). Current concepts for Anterior Cruciate Ligament reconstruction: A Criterion-Based Rehabilitation Progression. J Orthop Sport PHYS Ther [Internet]. 42(7):601-14. Available from: http://www.ncbi.nlm.nih.gov/pmc/articles/PMC3576892/pdf/nihms-440663.pdf

Arroyo, R., Martín, S, Martín, M., Mayoral, O. (2013). Programas de Fisioterapia tras reconstrucción del ligamento cruzado anterior. Revisión sistemática. Cuest Fisioter. 43(3):323-39.

Takahashr, K., Hayashi, M., Fujii, T., Kawamura, K., Ozaki, T. (2012). Early rehabilitation with weight-bearing standing-shaking-board exercise in combination with electrical muscle stimulation after anterior cruciate ligament reconstruction. Acta Med Okayama.66(3):231-7. Horschig, A., Neff, T., Serrano, A. (2014). Utilization of autoregulatory progressive resistance exercise in transitional rehabilitation periodization of a high school football-player following anterior cruciate ligament reconstruction: a case report. Int J Sports Phys Ther. 9(5):691-8.

Radice, F., Chamorro, C., Yañez, R., Vergara, F., González, F., Zelaya, G. (2010). Retorno deportivo en atletas de alto rendimiento después de reconstrucción de Ligamento Cruzado Anterior de Rodilla. Artroscopia.17(3):233-40.

Kinikli, G., Yüksel, I., Baltaci, G., Atay, Ö. (2014). The effect of progressive eccentric and concentric training on functional performance after autogenous hamstring anterior cruciate ligament reconstruction: A randomized controlled study. Acta Orthop Traumatol Turc. 48(3):283-9.

Meuffels, D., Poldervaart, M., Diercks, R., Fievez, A., Patt, T., Hart, C., et al. (2012). Guideline on anterior cruciate ligament injury. Acta Orthop. 2012;83(4):379-86. 